\title{
"PROSPECT THEORY" E A VELOCIDADE IDEAL DA LIBERALIZAÇÃO ECONÓMICA: UMA ANÁLISE CRÍTICA DAS CONCLUSÕES DO BANCO MUNDIAL
}

\author{
Luis G. Bernardes*
}

\begin{abstract}
Um recente estudo do Banco Mundial, baseado na transição económica que ocorre no Leste Europeu, conclui que a "terapia de choque" é a estratégia ideal de liberalização e reprova a política dos países que preferiram o gradualismo. Neste artigo, eu baseio-me num trabalho teórico anterior, em que discuti este topico face a um modelo alternativo de comportamento chamado "prospect theory", para mostrar como o estudo empírico do Banco Mundial é impreciso. Os dados disponíveis que apoiam as conclusões desse meu trabalho sugerem que motivos de eficiência económica podem explicar a preferência por reformas graduais verificada nalguns países.
\end{abstract}

\section{INTRODUÇÃO}

As recentes transformações ocorridas no Leste Europeu vieram renovar o debate sobre a velocidade ideal da liberalização económica. Neste tópico, a referência neo-clássica continua a ser Mussa $(1982,1986)$ : se os agentes económicos forem racionais e não existirem distorções na mobilidade dos factores produtivos, uma estratégia tipo "terapia de choque" maximizará o crescimento económico, mesmo que haja custos de ajustamento. Por outras palavras, a política a seguir é eliminar imediatamente as distorções e deixar que o ajustamento dos factores se processe ao ritmo ideal de mercado. $\mathrm{Na}$ prática, contudo, vários têm sido os governos (incluindo alguns do Leste

\footnotetext{
* Professor Auxiliar, Universidade Católica Portuguesa - IUDPS (Viseu).
} 
Europeu) a preferir uma liberalização gradual, especialmente quando se trata de reformas microeconómicas como a redução de tarifas ou a liberalização de preços. Em função da análise feita por Mussa, argumentos a favor da liberalização gradual têm enfrentado algum cepticismo, especialmente porque nenhuma relação evidente foi estabelecida entre esses argumentos e o comportamento dos governos'. Alguns desses argumentos baseiam-se na irracionalidade dos agentes ou em distorções no processo de ajustamento causadas, por exemplo, por externalidades do tipo informacional ou de congestão (ver, entre outros, Gavin, 1993). Contudo, esta perspectiva assume implicitamente que os factores produtivos se movem a uma velocidade superior àquela que seria socialmente optima - uma hipótese que a evidência empírica parece contradizer ${ }^{2}$. Argumentos com um carácter mais próximo da economia política têm também sido apresentados. Baseiam-se em motivos como limitações orçamentais (Dewatripont \& Roland, 1992), problemas de credibilidade (Calvo, 1989), e preocupações redistributivas ou de carácter político (Mussa, 1986, Wei, 1993, Desai, 1995, 1997, e Dehejia, 1996 , são exemplos importantes) ${ }^{3}$. O problema com estes modelos tem sido o seu carácter bastante específico e, por vezes, a sua excessiva complexidade. Para além disso, recentes estudos empíricos parecem apoiar também o uso da "terapia de choque". Por exemplo, o Banco Mundial (1996) e Fischer et al. (1996a, 1996b), baseando-se na experiência do Leste Europeu, concluem categoricamente que, quanto mais rápida for a liberalização, maior a taxa de crescimento económico ${ }^{4}$.

Noutro local (Bernardes, 1998), questionei as conclusões neoclássicas nesta matéria e propus um novo argumento (parcial) a favor do gradualismo. Baseei-me, especificamente, na "prospect theory" de comportamento desenvolvida por Kahneman e Tversky ${ }^{5}$ para mostrar que a análise neoclássica não é necessariamente correcta, uma vez que depende de hipóteses específicas sobre as preferências dos indivíduos. Mais ainda, provei que, se os agentes se comportarem de acordo com a "prospect theory" e se não existirem distorções na sua mobilidade, uma estratégia gradual pode maximizar o crescimento económico, desde que a reforma implique uma perca de rendimento suficientemente grande para os trabalhadores do sector liberalizado. No presente artigo, mostrarei como os estudos empíricos que mencionei atrás são imprecisos e apresentarei dados preliminares que apoiam as minhas conclusões e sugerem que a eficiência económica pode explicar a preferência por reformas graduais, verificada nalguns países. 


\section{BREVE SÍNTESE TEÓRICA}

O modelo teórico que usei (1998) é um modelo convencional de ajustamento com incerteza, onde parâmetros e preços são perfeitamente conhecidos. As preferências individuais, contudo, são aquelas postuladas pela "prospect theory". Esta teoria tem sido vasta e positivamente testada e, apesar de não convencional, é aceite como uma referência sólida e importante no estudo do comportamento dos indivíduos ${ }^{6}$. As principais conclusões desse meu estudo assentaram, sobretudo, em três axiomas fundamentais da "prospect theory":

- os indivíduos tomam decisões em função de um ponto-de-referência (i.e., as pessoas analisam as alternativas em termos de ganhos e percas relativamente a um dado valor de referência, que é normalmente o "status-quo");

- os indivíduos dão maior peso a uma possível perca do que a um ganho equivalente;

- os indivíduos são avessos ao risco quando se trata de analisar potenciais ganhos, mas amantes do risco quando se trata de avaliar potenciais percas.

A partir destes axiomas, podemos caracterizar a (nova) função de "utilidade" do indíviduo - aquilo a que Kahneman e Tversky chamam "value function" - como um S assimétrico (ver figura 1), onde v(ganhos) representa a avaliação subjectiva que um indivíduo faz de um ganho e $\mathrm{v}$ (percas) é o seu equivalente para as percas.

\section{Figura 1}

HIPOTÉTICA "VALUE FUNCTION" DEFINIDA PARA DOIS PONTOS-DE-REFERENCIA DIFERENTES $\left(r_{0}\right.$ e $\left.r_{1}\right)$, COM $r_{1}>r_{0}$

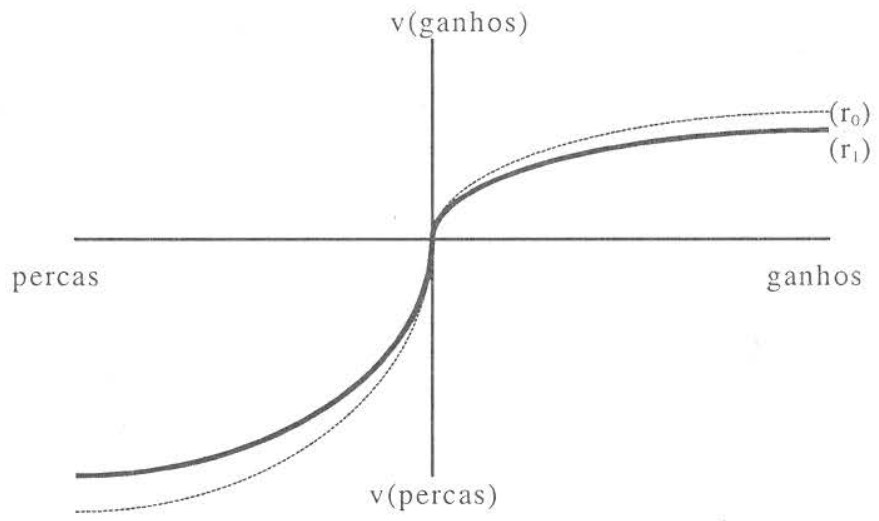


Com base nestes axiomas e nesta nova representação da "utilidade" dos indivíduos, pode provar-se que o mesmo problema de decisão é susceptível de levar um indivíduo a tomar atitudes diferentes, dependendo do seu ponto-de-referência - uma inconsistência comportamental que contraria os axiomas neoclássicos. Sendo assim, e de acordo com a "prospect theory", se durante o processo de liberalização for permitido aos indivíduos modificarem o seu ponto-de-referência, a análise neoclássica passará a estar incompleta, mesmo que usemos medidas tradicionais - como o crescimento económico - para avaliar o impacto da reforma. E, na verdade, eu provo que, se as pessoas se comportarem de acordo com a "prospect theory", uma liberalização gradual pode maximizar o crescimento, quando essa liberalização causa uma perca suficientemente grande de rendimento aos indivíduos empregues no sector liberalizado.

A justificação para este resultado deve ser dividida em duas partes. Primeiro, uma vez que os trabalhadores avaliam assimetricamente ganhos e percas, a velocidade a que se processará a sua mobilidade perante uma reforma tipo "terapia de choque" não é a socialmente óptima. Segundo, se os indíviduos se transferirem de sector a um ritmo superior ao socialmente óptimo, o problema é trivial e as conclusões neoclássicas são válidas. Contudo, o mesmo não é verdade se os trabalhadores forem lentos no seu processo de ajustamento - o caso mais previsível. Este resultado deve-se à sensibilidade marginal decrescente que as pessoas têm em relação às variações de rendimento à medida que estas aumentam de valor. Por outras palavras, à medida que o valor das alternativas a avaliar se afasta do ponto-de-referência ou da experiência dos indivíduos, estes têm uma dificuldade cada vez maior em interpretar pequenas mudanças nesse valor - um resultado que justifica a forma em " $S$ " da "value function". Por essa razão, quando a liberalização envolve uma perca de rendimento suficientemente grande, uma estratégia radical criará incentivos de ajustamento, aos quais os trabalhadores serão, na margem, insensíveis. Em consequência, uma estratégia gradual que permita aos indivíduos mudar o seu ponto-de-referência pode aumentar os estímulos de mobilidade, uma vez que "substituirá" a acumulação improdutiva de incentivos baseados em percas por incentivos baseados em ganhos. Em termos mais intuitivos, uma reforma gradual irá permitir aos indivíduos "conhecer" melhor as consequências da reforma enquanto esta se desenvolve, maximizando a sua resposta à mesma. Quão eficiente será a estratégia gradual (ou quão grande a perca precisa de ser) depende (também) do diferencial inicial de salários entre sectores. De facto, a aversão ao risco no domínio dos ganhos sugere que, quanto menor for a disparidade de rendimentos, maior o sucesso de uma estratégia gradual, 
uma vez que isso permitirá aos trabalhadores infortunados aperceber-se mais claramente da sua situação de perdedores.

\section{ANÁLISE EMPÍRICA}

Nesta secção, e concretizando o objectivo principal deste artigo, proponho-me discutir os resultados teóricos que atrás descrevi no contexto da transição económica do Leste Europeu. Ao mesmo tempo, pretendo questionar os resultados apresentados pelo Banco Mundial que, usando os mesmos dados em que me basearei, concluiu que existe uma relação inequivocamente positiva entre a rapidez da liberalização e o crescimento económico, advogando por isso o recurso à "terapia de choque".

Para analisar o meu resultado principal - uma estratégia gradual pode maximizar o crescimento económico, quando a reforma implique uma perca suficientemente grande de rendimento aos indivíduos empregues no sector liberalizado (ou, para usar a definição teórica mais precisa, sempre que a reforma cause um aumento suficientemente grande, na desigualdade de rendimentos entre vencedores e perdedores) que casos uma reforma leva a essa "perca suficientemente grande de rendimento". Claramente, este é um aspecto não trivial. Contudo, considerando que os países de menor rendimento são aqueles potencialmente menos preparados (económica e socialmente) para assistir aos trabalhadores e às indústrias afectadas - um pressuposto que eu defendo a seguir -, uma hipótese plausível é que, permanecendo tudo o resto igual, aqueles serão os países onde uma dada reforma é mais passível de gerar essa "perca suficientemente grande de rendimento" aos trabalhadores afectados. Três razões servem para justificar este meu argumento: (1) as já deterioradas condições socioeconómicas nesses países - o que significa também "pequenos" mercados e pouca capacidade financeira - tornarão mais difícil às indústrias afectadas proteger os seus trabalhadores; (2) nesses mesmos países, é menos provável que os trabalhadores afectados tenham fontes alternativas de rendimento; (3) certamente, faltarão aos governos e a outras instituições os recursos sociais e económicos necessários à implementação das habituais medidas compensatórias, destinadas a ajudar trabalhadores e empresas. Se este argumento estiver correcto, isso significa, de acordo com a "prospect theory", que a terapia de choque só é a estratégia ideal para países de maior rendimento. Em países de menor rendimento, uma reforma gradual poderá produzir melhores resultados. 
Assim, baseando-me na transição do Leste Europeu, mais especificamente em dados produzidos em 26 países entre 1989 e 1995, as duas hipóteses que começo por testar são:

I) Para países de maior rendimento, a relação entre a velocidade da liberalização e a taxa de crescimento é inequivocamente positiva.

II) Para países de menor rendimento, uma reforma mais lenta pode produzir melhores resultados que uma estratégia tipo "terapia de choque". Por isso, a relação entre a velocidade da reforma e o crescimento económico não será tão claramente positiva, podendo mesmo ser negativa.

Para definir que países são de "maior rendimento" e de "menor rendimento", baseei-me no seu PNB "per capita" de 1998, medido em termos de PPP (paridade de poder de compra) ${ }^{7}$. A escolha do valor (rendimento) de separação reflecte o nosso conhecimento das economias em causa. Contudo, é importante notar que os resultados obtidos são robustos a mudanças neste valor de separação - uma situação que confirmei repetindo as mesmas regressões que apresento neste artigo mas usando um maior/menor rendimento de separação para o grupo de maior/menor rendimento, respectivamente (ver anexo' 1.A para uma descrição destes grupos, e para fontes e critérios usados).

\section{TESTES PRINCIPAIS}

\subsection{Um Teste Preliminar}

Baseando-se na transição do Leste Europeu, o Banco Mundial, no seu Relatório de 1996, concluiu que existe uma relação positiva e inequívoca entre a velocidade da liberalização e o crescimento económico. Nesse estudo, - Banco Mundial usou como variável dependente a taxa média de crescimento corrigida do PIB para os anos de 1989 a 1995 (GDPgr) e como variável explicativa um índice de liberalização (SPEED) desenvolvido por De Melo et al. (1996) e que assume valores de 1 a 8 , sendo 8 o equivalente à velocidade máxima de liberalização 9 . A relação estimada pelo Banco Mundial é reproduzida na figura $2 \mathrm{a}^{10}$ e os seus parâmetros detalhados na Tabela 1, coluna (1). A conclusão é que um ponto extra de velocidade na escala definida pelo Banco Mundial equivaleria a um aumento na taxa de crescimento anual do PIB igual a $1.55 \%$. 
Figura 2a

A RAPIDEZ DA LIBERALIZAÇÃO E O CRESCIMENTO ECONÓMICO (BANCO MUNDIAL)

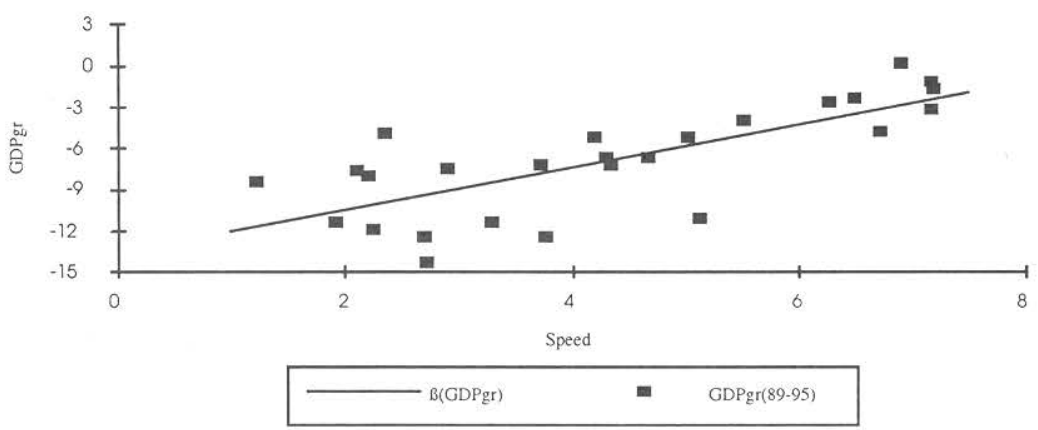

Tabela 1

CRESCIMENTO ECONÓMICO E VELOCIDADE DE LIBERALIZAÇÃO (I)

\begin{tabular}{|c|c|c|c|c|c|}
\hline & \multirow{2}{*}{$\begin{array}{c}\text { Modelo BM } \\
\text { (1) }\end{array}$} & \multicolumn{4}{|c|}{ Modelo "Prospect Theory" } \\
\hline & & (2) & (3) & (4) & (5) \\
\hline CONST & $\begin{array}{l}-13.54 * * \\
(-10.24)\end{array}$ & $\begin{array}{c}-16.17 * * \\
(-7.61)\end{array}$ & $\begin{array}{l}-10.38 * * \\
(-3.12)\end{array}$ & $\begin{array}{l}-8.87 * * \\
(-3.23)\end{array}$ & $\begin{array}{l}-7.99 * * \\
(-2.53)\end{array}$ \\
\hline SPEED & $\begin{array}{l}1.55^{* *} \\
(5.52)\end{array}$ & $\begin{array}{l}2.04 * * \\
(5.25)\end{array}$ & $\begin{array}{l}1.26 * * \\
(2.45)\end{array}$ & $\begin{array}{l}1.39 * * \\
(3.73)\end{array}$ & $\begin{array}{l}1.22 * * \\
(2.59)\end{array}$ \\
\hline DLOW & & $\begin{array}{c}5.33 * \\
(1.87)\end{array}$ & $\begin{array}{l}4.84 * \\
(1.83)\end{array}$ & $\begin{array}{l}6.62 * * \\
(2.67)\end{array}$ & $\begin{array}{l}6.63 * * \\
(2.44)\end{array}$ \\
\hline SPEED*DLOW & & $\begin{array}{l}-1.27^{*} \\
(-1.93)\end{array}$ & $\begin{array}{l}-1.39 * * \\
(-2.27)\end{array}$ & $\begin{array}{l}-1.37 * \\
(-1.92)\end{array}$ & $\begin{array}{l}-1.52 * \\
(-1.98)\end{array}$ \\
\hline DFSU & & & $\begin{array}{l}-3.45 * * \\
(-2.15)\end{array}$ & & $\begin{array}{l}-1.14 \\
(-0.59)\end{array}$ \\
\hline AGR89 & & & & $\begin{array}{l}-0.24 * * \\
(-3.45)\end{array}$ & $\begin{array}{l}-0.21 * * \\
(-2.26)\end{array}$ \\
\hline Amostra & 26 & 26 & 26 & 25 & 25 \\
\hline $\mathrm{R}^{2}$ & 0.56 & 0.62 & 0.69 & 0.76 & 0.77 \\
\hline
\end{tabular}

Notas: A variável dependente é GDPgr; t-statistics em parêntesis.

* significativo a $10 \%$; * significativo a $5 \%$ 
Contudo, como escrevi, o modelo teórico baseado na "prospect theory" prevê que o declive desta relação para os países de menor rendimento seja significativamente menor do que aquele calculado para os países de maior rendimento, podendo mesmo ser negativo. Sendo assim, como estudo preliminar das minhas hipóteses, decidi testar a robustez das conclusões do Banco Mundial à divisão da sua amostra em países de maior e menor rendimento. Para isso, recorro às mesmas variáveis usadas pelo Banco Mundial mas, agora, calculando coeficientes de estimação diferentes consoante o nível de rendimento dos países. A equação a estimar é, portanto:

$$
G D P g r=\beta_{0}+\beta_{1} S P E E D+\beta_{2} D L O W+\beta_{3} S P E E D * D L O W+\varepsilon
$$

onde DLOW é uma variável "dummy" que assume o valor " 1 " para países de menor rendimento e "0" para países de maior rendimento; $\beta_{1}$ é o declive da relação entre velocidade e crescimento para os países de maior rendimento; $\beta_{1}+\beta_{3}$ é o declive dessa mesma relação para países de menor rendimento; e $\beta_{3}$ indica-nos quão menor é o declive desta relação para os países de menor rendimento. Os resultados da estimação feita, usando o Método dos Mínimos Quadrados, são apresentados na Tabela 1, coluna (2), e representados na figura $\mathbf{2} \mathbf{b}$.

Figura 2b

A RAPIDEZ DA LIBERALIZAÇÃO E O CRESCIMENTO ECONÓMICO (MODELO ALTERNATIVO)

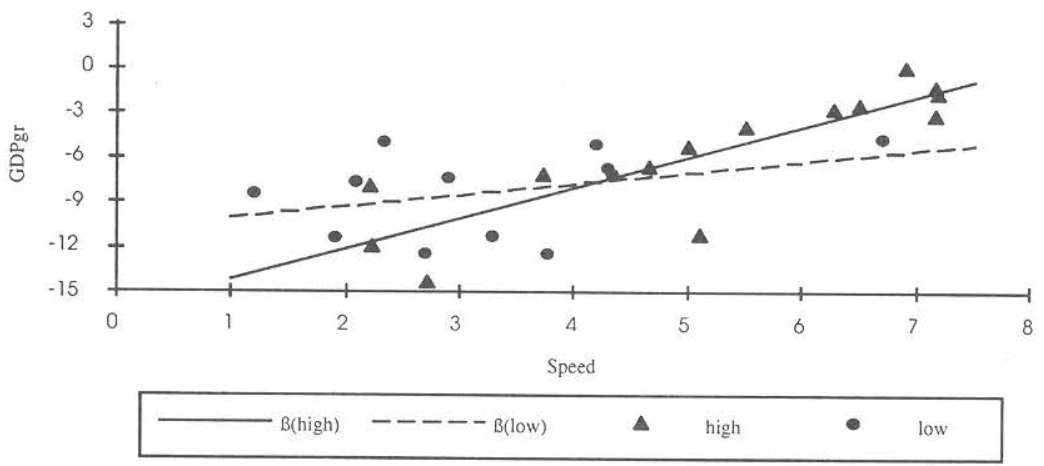

Embora os declives estimados sejam ambos positivos, verifica-se que o declive desta relação para os países de menor rendimento - um ponto extra 
de velocidade equivale a um aumento na taxa de crescimento igual a $0.77 \%$ - é consideravelmente menor do que para os países de maior rendimento, nos quais um ponto extra de velocidade permite um acréscimo na taxa de crescimento de $2.04 \%$. Mais importante, esta diferença de declives é estatisticamente significativa. Para além disso, devo notar que a natureza destas conclusões seria ainda mais robusta se excluíssemos da amostra a Macedónia (representada na figura $2 \mathrm{~b}$ pelo círculo correspondente a um índice de velocidade igual a 6.71$)^{11}$. Isto é particularmente importante uma vez que as estatísticas disponíveis para a Macedónia estão entre as de menor significado e confiança, devido aos conflitos internos que afectaram esse país. Estes resultados indicam, para já, que as conclusões do Banco Mundial não são tão inequívocas ou gerais como é proposto no seu relatório. A seguir, incluo neste estudo alguns outros parâmetros que a análise teórica sugere como relevantes. O principal resultado, contrariando as conclusões do Banco Mundial, é o de que em países de menor rendimento a relação entre a velocidade da liberalização e o crescimento económico pode mesmo ser negativa.

\subsection{Um teste mais completo}

De acordo com o modelo baseado na "prospect theory", pelo menos dois outros factores contribuem decisivamente para determinar a velocidade ideal da liberalização: os parâmetros de comportamento - particularmente, os aspectos sociopolíticos que envolvem a reforma em cada país e que podem influenciar o comportamento e as mudanças do ponto-de-referência dos indivíduos - e os custos de ajustamento ou transição. Obviamente, neste quadro, poder-se-ia argumentar a favor da inclusão de inúmeras variáveis nesta análise. Contudo, o pequeno número de observações disponíveis e outras limitações ao nível dos dados recomendam uma atitude mais prudente e circunscrita.

Assim, de forma a ter em conta a heterogeneidade sociopolítica dos países - nomeadamente as diferentes condições sociais, herança política e influências geopolíticas, sob as quais os processos de liberalização foram iniciados - incluo na análise uma variável "dummy" regional (DFSU) que servirá para distinguir dados provenientes da Antiga União Soviética (URSS) daqueles provenientes dos restantes países ${ }^{12}$. Quanto aos custos de transição, um conceito habitual é que o choque inicial resultante do processo de ajustamento é maior nas economias onde o sector industrial tem maior peso, dada a rigidez e ineficiências já instaladas. Para levar em conta esta possibilidade, incluo nos testes empíricos o "share" inicial da produção 
agrícola relativo ao PIB total, para cada país (AGR89). A Tabela 1, colunas (3) a (5), apresenta os resultados das regressões efectuadas incluindo já estas variáveis.

Apesar do número limitado de observações e da natureza incompleta, de curto prazo e até circunstancial dos dados disponíveis, os resultados apresentam-se consistentes com as minhas hipóteses e sugerem que a relação entre a velocidade da reforma e o crescimento económico depende do nível inicial de rendimento de cada país. Para o grupo de maior rendimento, a correlação entre a velocidade e o crescimento - dada por $\hat{\beta}($ SPEED) - é inequívoca e significativamente positiva. Este resultado confirma a minha primeira hipótese e a superioridade das estratégias tipo "terapia de choque" nestes países. Para os países incluídos no grupo de menor rendimento, as conclusões são bem diferentes. Em primeiro lugar, a diferença estimada de declives entre os dois grupos - dada por $\hat{\beta}\left(S P E E D^{*} D L O W\right)$ - é sistematicamente negativa e significativamente diferente de zero em todas as regressões baseadas no modelo alternativo [colunas (2) a (5)]. Isto vem confirmar que $o$ declive da relação entre a velocidade e $o$ crescimento assume um valor menos pronunciado em países de menor rendimento. Em segundo lugar, verificamos que, nas regressões que incluem a variável "dummy" DFSU [colunas (3) e (5)], o declive absoluto dessa relação - dado por $\hat{\beta}($ SPEED $)+\hat{\beta}(S P E E D * D L O W)$ - é mesmo negativo (igual a -0.13 e a -0.3 , respectivamente), sendo virtualmente igual a zero na regressão (4). Estes resultados confirmam a minha segunda hipótese, sugerindo que a "terapia de choque" não é a política eficiente em países de menor rendimento.

Quanto à variável AGR89 (que representa o peso da agricultura na actividade económica do país), o Banco Mundial escreve que o PIB tem aumentado mais ou diminuído menos, desde 1989 , nos países mais pobres e mais agrícolas do que nos países mais ricos com maiores sectores industriais. Isto vem ao encontro da crença, que referi atrás, de que em países com maior peso industrial os custos iniciais de ajustamento são maiores. As minhas regressões, contudo, não confirmam esta afirmação do Banco Mundial, como se pode atestar pelos coeficientes negativos associados à variável AGR89 [colunas (4) e (5), na Tabela 1]. Para além de possíveis imperfeições nos dados, esta conclusão pode ser explicada por circunstâncias referidas em De Melo et al. (1996), como a degradação do preço relativo dos bens agrícolas, a falta de liquidez no sector agrícola, ou a seca que afectou a generalidade dos países do Leste Europeu no início dos anos 90. Baseando-me numa perspectiva mais próxima do meu modelo teórico, outra possível explicação é o período mais longo de adaptação e educação necessários para completar a transição económica em países onde a força de trabalho é predominantemente agrícola. 


\section{ANÁLISE DE SENSIBILIDADE}

\subsection{Uma medida não corrigida de crescimento}

Como se disse, a medida de crescimento (GDPgr) usada nos estudos do Banco Mundial é uma medida corrigida, de forma a ter em conta os efeitos das perturbações sociais ocorridas nalguns países e, por exemplo, as suas diferenças em termos de recursos naturais. É possível, por isso, que alguns dos resultados que reportei atrás possam estar enviezados pelas correcções feitas pelo Banco Mundial a essa variável. Assim, para confirmar a robustez das minhas conclusões, repeti os testes anteriores mas usando como variável dependente uma medida não corrigida de crescimento: o valor do PIB em 1995 relativo a 1989 (OUTP). Para controlar os efeitos dos conflitos internos, incluí ainda uma variável "dummy" que assume o valor " 1 " para países onde se verificaram esses conflitos. ${ }^{13}$ Os resultados destes novos testes, embora estatisticamente menos robustos, são idênticos àqueles já reportados, confirmando, assim, as minhas conclusões iniciais (ver Tabela 2).

Tabela 2

CRESCIMENTO ECONÓMICO E VELOCIDADE DE LIBERALIZAÇÃO (II)

\begin{tabular}{cccccc}
\hline & Modelo BM & \multicolumn{4}{c}{ Modelo "Prospect Theory" } \\
& \multicolumn{1}{c}{$(1)$} & $(2)$ & \multicolumn{1}{c}{$(3)$} & $4)$ & \multicolumn{1}{c}{$(5)$} \\
\hline CONST & $38.88^{* *}$ & 19.8 & $70.74^{* *}$ & $64.5^{* *}$ & $78.25^{* *}$ \\
& $(5.03)$ & $(1.69)$ & $(4.96)$ & $(4.17)$ & $(5.17)$ \\
SPEED & $6.38^{* *}$ & $9.45^{* *}$ & 2.51 & $5.48^{* *}$ & 2.56 \\
& $(4.06)$ & $(4.44)$ & $(1.14)$ & $(2.72)$ & $(1.16)$ \\
DLOW & & $29.92^{*}$ & $25.5^{* *}$ & $32.2^{* *}$ & $30.03^{* *}$ \\
& & $(1.92)$ & $(2.25)$ & $(2.2)$ & $(2.27)$ \\
SPEED*DLOW & & -5.01 & $-6.14^{* *}$ & -3.33 & -5.68 \\
& & $(-1.4)$ & $(-2.35)$ & $(-0.86)$ & $(-1.56)$ \\
DFSU & & & $-30.56^{* *}$ & & $-21.52^{* *}$ \\
& & & $(-4.44)$ & & $(-2.32)$ \\
AGR89 & & & & $-1.43^{* *}$ & -0.72 \\
& & & & $(-3.67)$ & $(-1.54)$ \\
\hline Amostra & 26 & 26 & 26 & 25 & 25 \\
$\mathrm{R}^{2}$ & 0.57 & 0.64 & 0.82 & 0.79 & 0.84 \\
\hline
\end{tabular}

Notas: A variável dependente é OUTP; t-statistics em parêntesis.

* significativo a $10 \%$; ** significativo a $5 \%$. 


\subsection{Uma medida diferente de velocidade}

Uma dúvida importante em relação aos testes que já descrevi é a natureza contínua do índice de liberalização que tenho vindo a usar. Na verdade, o índice de liberalização proposto pelo Banco Mundial (SPEED) não reflecte com exactidão o conceito discreto de gradualismo e "terapia de choque" que está por trás dos resultados teóricos do meu modelo. Por esse motivo, decidi testar mais uma vez as hipóteses iniciais, mas agora usando como principal variável explicativa uma variável "dummy" que assume o valor "1" para países não gradualistas e "0" para países gradualistas. A equação a estimar é:

$$
G D P g r=\alpha_{0}+\alpha_{1} N G R D+\alpha_{2} D L O W+\alpha_{3} N G R D * D L O W+v
$$

onde $\alpha_{1}$ mede o crescimento económico dos países não-gradualistas de maior rendimento relativo aos outros países de maior rendimento; $\alpha_{1}+\alpha_{3}$ tem função idêntica mas para os países de menor rendimento; e $\alpha_{3}$ mede a diferença na "performance" relativa dos países não-gradualistas, consoante o grupo de rendimento a que pertencem.

Dado o carácter particular que as definições de gradualismo e de "terapia de choque" assumem no modelo teórico baseado na "prospect theory", é particularmente difícil definir que países têm preferido uma estratégia gradual. Assim, para evitar considerações demasiado subjectivas, usarei como possíveis "cut-off points" ou valores-limite os sugeridos pela divisão, em quatro grupos, feita pelo Banco Mundial para caracterizar países em função da velocidade da sua liberalização ${ }^{14}$. Isso quer dizer que, em termos da variável SPEED, os valores-limite que usarei são $2.5,4$ e 6 , respectivamente. Os resultados das minhas estimativas para estes valores são apresentados na Tabela 3, e, mais uma vez, são consistentes com as hipóteses testadas. 
Tabela 3

CRESCIMENTO E GRADUALISMO

\begin{tabular}{|c|c|c|c|c|c|c|}
\hline & \multicolumn{2}{|c|}{ NGRD=1 se Speed $=2.5$} & \multicolumn{2}{|c|}{ NGRD=1 se Speed $=4$} & \multicolumn{2}{|c|}{ NGRD $=1$ se Speed $=6$} \\
\hline & (1) & (2) & (3) & (4) & (5) & (6) \\
\hline CONST & $\begin{array}{l}-9.91^{* *} \\
(-3.97)\end{array}$ & $\begin{array}{l}-4.13^{*} \\
(-1.94)\end{array}$ & $\begin{array}{r}-10.3^{* *} \\
(-7.09)\end{array}$ & $\begin{array}{l}-5.38^{* *} \\
(-2.49)\end{array}$ & $\begin{array}{l}-8.49^{* *} \\
(-8.51)\end{array}$ & $\begin{array}{l}-5.54^{* *} \\
(-3.49)\end{array}$ \\
\hline NGRD & $\begin{array}{c}5.0^{*} \\
(1.78)\end{array}$ & $\begin{array}{c}1.63 \\
(0.79)\end{array}$ & $\begin{array}{l}6.56 * * \\
(3.81)\end{array}$ & $\begin{array}{l}3.11 \\
(1.61)\end{array}$ & $\begin{array}{l}6.7 * * \\
(4.4)\end{array}$ & $\begin{array}{c}3.75^{*} \\
(1.97)\end{array}$ \\
\hline DLOW & $\begin{array}{c}1.86 \\
(0.58)\end{array}$ & $\begin{array}{c}1.86 \\
(0.84)\end{array}$ & $\begin{array}{c}0.84 \\
(0.47)\end{array}$ & $\begin{array}{c}0.84 \\
(0.54)\end{array}$ & $\begin{array}{l}-0.1 \\
(-0.08)\end{array}$ & $\begin{array}{l}-0.6 \\
(-0.49)\end{array}$ \\
\hline NGRD*DLOW & $\begin{array}{l}-5.33 \\
(-1.47)\end{array}$ & $\begin{array}{l}-4.85^{*} \\
(-1.95)\end{array}$ & $\begin{array}{l}-2.98 \\
(-1.21)\end{array}$ & $\begin{array}{l}-4.46^{*} \\
(-2.01)\end{array}$ & $\begin{array}{l}-2.84 \\
(-0.86)\end{array}$ & $\begin{array}{l}-2.35 \\
(-0.77)\end{array}$ \\
\hline DFSU & & $\begin{array}{l}-5.78^{* *} \\
(-5.04)\end{array}$ & & $\begin{array}{l}-4.92 * * \\
(-2.81)\end{array}$ & & $\begin{array}{l}-3.37 * * \\
(-2.28)\end{array}$ \\
\hline Amostra & 26 & 26 & 26 & 26 & 26 & 26 \\
\hline $\mathrm{R}^{2}$ & 0.23 & 0.65 & 0.52 & 0.65 & 0.55 & 0.64 \\
\hline
\end{tabular}

Notas: A variável dependente é GDPgr; t-statistics em parêntesis.

* significativo a $10 \% ; * *$ significativo a $5 \%$.

Como esperado, o coeficiente estimado da variável NGRD é positivo para todas as especificações, confirmando a "terapia de choque" como a estratégia ideal para os países de maior rendimento. Para os países de menor rendimento, a natureza das conclusões depende, em parte, do valor-limite escolhido. Para os valores-limite mais baixos [regressões (1) a (4)], a diferença estimada de declives (NGRD*DLOW) é sempre negativa (isto é, a "performance" relativa dos países não gradualistas de menor rendimento foi inferior à dos não gradualistas de maior rendimento) e, quando incluímos a "dummy" DFSU [colunas (2) e (4)], é também estatisticamente significativa. Mais ainda, com excepção da regressão (3), o declive absoluto da relação entre velocidade e crescimento $\left(\hat{\alpha}_{1}+\hat{\alpha}_{3}\right)$ é mesmo negativo, sugerindo, mais uma vez, que uma estratégia tipo "terapia de choque" não é a melhor para os países de menor rendimento. Para o valor-limite mais elevado [regressões (5) e (6)], os resultados são menos significativos. Um aspecto importante, contudo, é que neste caso existe apenas um país de menor rendimento (Macedónia), pertencente à amostra não gradualista. Isto torna as conclusões para o grupo de países de menor rendimento virtualmente irrelevantes - na verdade, para o valor-limite mais baixo, as conclusões devem padecer do mesmo mal, dado que nesse caso não abundam os países gradualistas de maior rendimento. Contudo, estas constatações acabam por fornecer ainda 
maior consistência às minhas hipóteses, uma vez que significam que o modelo teórico "previu" acertadamente a escolha de estratégia dos países. No seu conjunto, estas conclusões sugerem que, na "prospect theory", poderemos encontrar razões de eficiência económica para explicar a adopção de estratégias graduais nalguns países. Na verdade, como alguém já disse: "por vezes, a estrada menos caminhada é menos caminhada por um bom motivo."

\section{A IMPORTÂNCIA DA DESIGUALDADE DE RENDIMENTOS}

De acordo com os resultados téricos que apresentei no princípio deste artigo, pelo menos uma outra variável pode afectar a "performance" relativa dos países que adoptam uma estratégia gradual: a disparidade inicial de rendimentos entre os trabalhadores do sector "eficiente" e os do sector liberalizado. Especificamente, as minhas conclusões sugerem que, quanto maior for a desigualdade de rendimentos, 'menos eficiente é uma liberalização gradual.

Testar esta hipótese é particularmente difícil devido às grandes limitações estatísticas. Ainda assim, fazendo uso dos dados existentes, efectuo alguns testes que nos poderão dar uma ideia aproximada sobre a realidade desta hipótese.

Uma vez que não existe informação detalhada sobre as características do sector público e privado - aqueles em que, tendo em conta a filosofia do modelo teórico, eu dividiria a economia para fins deste estudo - em termos da sua produção, salários e força de trabalho, decidi recorrer ao coeficiente Gini como medida da desigualdade existente. Infelizmente, mesmo neste caso, alguns dos países que compõem a amostra não têm esta estatística e, muitos dos que a têm, têm-na apenas para anos mais recentes (1992 e 1993), enquanto a situação ideal seria ter estes valores para o início do período.

Para testar a minha hipótese, uso uma variável composta, obtida pela multiplicação da variável de desigualdade (GINI) por uma "dummy" (GRD) que assume o valor " 1 " para países gradualistas e " 0 " para os outros. $O$ critério que uso para classificar países gradualistas é o mesmo que usei na secção anterior. Contudo, devido à quase inexistência de dados para países gradualistas cuja rapidez de liberalização é inferior a 2.5 (o mais baixo valor-limite), limitar-me-ei aos outros dois valores-limite: 4 e 6 . De acordo com os resultados do modelo, o coeficiente estimado para GINI*GRD deverá assumir um valor negativo: i.e., entre países gradualistas, aqueles com 
menores índices de desigualdade serão também os que alcançaram maiores taxas de crescimento. Os resultados são apresentados na Tabela 4.

Tabela 4

CRESCIMENTO, VELOCIDADE E DESIGUALDADE

\begin{tabular}{ccccc}
\hline & \multicolumn{2}{c}{ GRD-1 se Speed - 4 } & \multicolumn{2}{c}{ GRD-1 se Speed - 6 } \\
\cline { 2 - 5 } & $(1)$ & $(2)$ & $(3)$ & $(4)$ \\
\hline \multirow{2}{*}{ CONST } & $-12.42^{* *}$ & $-5.58^{*}$ & $-7.45^{* *}$ & -5.11 \\
& $(-4.28)$ & $(-1.9)$ & $(-2.16)$ & $(-1.57)$ \\
SPEED & $1.46^{* *}$ & $0.82^{*}$ & 0.86 & $0.83^{*}$ \\
& $(3.0)$ & $(1.86)$ & $(1.69)$ & $(1.79)$ \\
DLOW & 3.38 & 4.79 & 3.12 & 5.19 \\
& $(1.03)$ & $(1.5)$ & $(1.13)$ & $(1.75)$ \\
SPEED*DLOW & -0.97 & -1.32 & -0.99 & -1.3 \\
& $(-1.37)$ & $(-1.67)$ & $(-1.63)$ & $(-1.5)$ \\
GINI*GRD & -0.06 & -0.02 & $-0.15 * *$ & -0.1 \\
& $(-1.09)$ & $(-0.49)$ & $(-2.47)$ & $(-1.24)$ \\
DFSU & & -1.43 & & 1.02 \\
& & $(-0.78)$ & & $(0.42)$ \\
AGR89 & & $-0.19 * *$ & & $-0.21^{* *}$ \\
& & $(-2.27)$ & & $(-2.5)$ \\
\hline Amostra & 22 & 21 & 20 & 19 \\
R ${ }^{2}$ & 0.68 & 0.84 & 0.78 & 0.87 \\
\hline
\end{tabular}

Notas: A variável dependente é GDPgr; t-statistics em parêntesis.

* significativo a $10 \%$; ** significativo a $5 \%$.

Apesar das limitações estatísticas que mencionei e que nos obrigam a encarar estas conclusões com alguma reserva, os resultados destes testes são consistentes com a minha hipotese. $\mathrm{Na}$ verdade, e como previsto, o coeficiente estimado de GINI*GRD é sistematicamente negativo. Os resultados são particularmente robustos quando usamos o valor-limite mais alto - regressões (3) e (4) - o que constitui uma situação normal dada a pequena amostra de países gradualistas usada nas regressões (1) e (2). Todas as outras principais implicações do modelo alternativo teórico são, também aqui, confirmadas. 


\section{CONCLUSÕES}

Noutro estudo (Bernardes, 1998), mostrei que, se os indivíduos se comportarem de acordo com a "prospect theory" de Kahneman e Tversky (1979), a velocidade óptima de liberalização depende das condições iniciais do país (ou da região ou do sector, conforme o destinatário da reforma), nomeadamente do grau das distorções existentes e do diferencial de rendimentos verificado entre os trabalhadores dos sectores eficiente $\mathrm{e}$ ineficiente. Especificamente, nesse trabalho, mostrei que uma liberalização gradual pode maximizar o crescimento económico, desde que a reforma implique uma perca de rendimento suficientemente grande para os trabalhadores do sector liberalizado. Quão grande precisa de ser essa perca ou quão efectiva será essa estratégia gradual dependem (também) da mencionada diferença inicial de rendimentos entre sectores.

No presente artigo, usei estes resultados para discutir a evidência fornecida pela liberalização que ocorre no Leste Europeu e para criticar as conclusões relativas a este assunto, apresentadas pelo Banco Mundial no seu relatório de 1996. Os dados preliminares que analisei são consistentes com as minhas hipóteses teóricas e sugerem que na "prospect theory" se poderão encontrar motivos de eficiência económica para explicar a adopção de estratégias graduais nalguns países.

\section{NOTAS}

${ }^{1}$ Rodrik (1995), Tommasi \& Velasco (1995) fornecem um tratamento detalhado destes assuntos. A discussão que proponho a seguir baseia-se sobretudo nesses trabalhos.

${ }^{2}$ De facto, argumentos a favor de uma liberalização ainda mais radical que a "terapia de choque" (overshooting) — como aqueles apresentados por Caplin \& Leahy (1993) e por Rodrik (1989) - são, neste contexto, ainda mais convincentes. Caplin \& Leahy (1993) concluem que overshooting pode ser necessário para compensar o déficit na criação de postos de trabalho causado por insuficiências de informação. Rodrik (1989) sugere a mesma solução mas como um instrumento de signaling para convencer os indivíduos acerca das intenções estratégicas do governo.

3 Alguns destes estudos dedicam-se, primeiramente, à questão da sequência da reforma. Ainda assim, os seus argumentos podem ser facilmente aplicados à questão da velocidade da liberalização.

${ }^{4}$ Desai (1997) questiona a metodologia usada nestes testes - ainda que não os resultados finais - e nota que a relação entre a rapidez da liberalização e outras variáveis económicas (por exemplo, a taxa de emprego) é negativa, podendo ter consequências futuras prejudiciais para o crescimento económico. 
${ }^{5}$ A referência original é Kahneman \& Tversky (1979). No seu trabalho, eles refutam a "expected utility theory" (teoria da utilidade esperada) como modelo descritivo do comportamento dos indivíduos em situações de risco, e propõem uma teoria alternativa a que chamaram "prospect theory".

${ }^{6}$ Bazerman (1998) classifica a "prospect theory" como "... the most important advance in our understanding of behavior decision making processes in the last twenty years". Esta teoria tem sido testada e aplicada em áreas tão importantes como Finanças e Gestão; Economia do Ambiente e da Energia; Direito Económico; Economia Agrícola; e comportamento do consumidor.

Dado o carácter incompleto das estatísticas disponíveis para o Leste Europeu, o uso de valores calculados a PPP é, ainda assim, o menos controverso. No entanto, o uso de outros indicadores disponíveis não acarretaria mudanças significativas nos resultados. De facto, repeti os testes que apresento neste artigo com pequenas variações na classificação dos países em termos do seu rendimento inicial, e as conclusões foram as mesmas.

${ }^{8}$ Esta variável é corrigida de forma a ter em conta factores exógenos como tensões regionais e diferenças no nível inicial de rendimento. Para uma explicação mais detalhada ver Banco Mundial (1996), fig. 2.2, p. 28.

${ }^{9}$ Ver Banco Mundial (1996), fig. 1.2, p. 14, e De Melo et al. (1996), para uma descrição detalhada deste índice. Fischer $e$ t al. (1996) também se baseiam nesta variável para concluir que existe uma relação positiva entre velocidade de reforma e crescimento.

${ }^{10}$ Esta figura reproduz parcialmente a figura $2.2 \mathrm{em}$ Banco Mundial (1996).

${ }^{11}$ Para o mesmo $\hat{\beta}(h i g h)=2.04$, o valor de $\hat{\beta}_{3}$ (o diferencial estimado de declives entre os dois grupos de rendimento) $\hat{e}-1.265$ com a Macedónia e -1.56 sem a Macedónia.

${ }^{12}$ Ver Banco Mundial (1996), De Melo et al. (1996) e Murrell (1996), para uma breve descrição das diferentes condições que rodearam o início das reformas nestas duas regiōes.

${ }^{13}$ Arménia, Azerbaijão, Croácia, Geórgia, Macedónia e Tajiquistão, de acordo com o critério do Banco Mundial.

${ }^{14}$ Ver Banco Mundial (1996), fig. 1.2, pág. 14. 


\section{Anexo: Apêndice estatístico}

\section{A. Ranking de Países (PNB per Capita a PPP, US\$ 1988)}

GRUPO DE MAIOR RENDIMENTO: Eslovénia, Estónia (FSU), Letónia (FSU), Rússia (FSU), Bielorrússia (FSU), Lituânia (FSU), Hungria, Geórgia (FSU), Bulgária, Ucrânia (FSU), Polónia + República Checa, República Eslovaca e Croácia.

GRUPO DE MENOR RENDIMENTO: Arménia (FSU), Cazaquistão (FSU), Moldávia (FSU), Azerbeijão (FSU), Turquemenistão (FSU), Roménia, República Kyrgyz (FSU), Uzbequistão (FSU), Tajiquistão (FSU), Albânia + Mongólia e FYR Macedónia.

Fonte: Fischer et al. (1996a). O valor-limite é 4940 US\$. A inclusão da Croácia e das Repúblicas Checa e Eslovaca no grupo de maiorrendimento, e da Mongólia e FYR Macedónia no grupo de menor rendimento, é baseada em dados do Banco Mundial para o PIB de 1989, medido a preços correntes.

\section{B. Variáveis e Fontes}

GDPgr: taxa média anual de crescimento do PIB de 1989 a 1995, corrigida pelos efeitos dos conflitos internos verificados nalguns países e pelas diferenças iniciais de recursos naturais e rendimento per capita. Fontes: Banco Mundial (1996) e De Melo et al. (1996).

OUTP: Outup total para $1995(1989=100)$. Fontes: Fischer et al. $(1996 \mathrm{a}$, 1996b).

SPEED: índice da velocidade de liberalização calculado como média ponderada da velocidade de liberalização das transações domésticas, das transações com o exterior, e da entrada de novas firmas. Fontes: Banco Mundial (1996) e De Melo et al. (1996).

DLOW: "dummy" classificativa dos países por rendimento; assume o valor "1" para os países de menor rendimento.

DFSU: "dummy" regional, que assume o valor "1" para países que pertenciam à antiga União Soviética.

AGR89: valor acrescentado agrícola em percentagem do PIB total para 1989. Fonte principal: World Development Indicators 97 - versão CD ROM (Banco Mundial). Valores para a Moldávia, Croácia, Eslovénia e Cazaquistão foram calculados com base também em De Melo et al. (1996). 
NGRD: "dummy" da velocidade de liberalização que assume o valor "1" para países não-gradualistas - para critério, ver texto principal.

GRD: "dummy" da velocidade de liberalização que assume o valor " 1 " para países gradualistas - para critério, ver texto principal.

GINI: Valor do coeficiente Gini medido em termos percentuais (1988-92). Fonte principal: Deininger \& Squire (1996) dataset. Outras fontes: Banco Mundial (1996) para Rússia, Estónia e República Kyrgyz (table 4.1, Goskmostat values), e Eslovénia (table 4.1). Sempre que mais de um valor estava disponível, o escolhido foi aquele obtido mais perto do início do período de análise (1989). 


\section{BIBLIOGRAFIA}

BANCO MUNDIAL (1995), World*Data 95 - versão CD ROM, Washington, D. C.

BANCO MUNDIAL (1996), World Development Report 1996: From Plan to Market, Washington, D. C.

BANCO MUNDIAL (1997), World Development Indicators 97 - versão CD ROM, Washington, D. C.

BERNARDES, L. (1998), "Prospect Theory and the Ideal Speed of Economic Liberalization: A Theoretical Analysis", mimeog., Columbia University.

CAPLIN, A. and J. LEAHY (1993), "The Economics of Adjustment," NBER Working Paper 4687.

De MELO, M., C. DENIZER e A. GELB (1996), "From Plan to Market, Patterns of Transition," Policy Research Working Paper 1564, Banco Mundial, Janeiro.

DEHEJIA, V. (1996), "Shock-Therapy vs. Gradualism: A Neoclassical Perspective," Eastern Economic Journal, 22 (4).

DEININGER, K. e L. SQUIRE (1996), “A New Data Set Measuring Income Inequality”, The World Bank Economic Review, 10

DESAI, P. (1995), “Beyond Shock-Therapy," Journal of Democracy, Vol. 6, No. 2.

DESAI, P. (1997), Going Global: Transition from Plan to Market in the World Economy, MIT Press.

DEWATRIPONT, M. and G. ROLAND (1992), "The Virtues of Gradualism and Legitimacy in the Transition to a Market Economy," Economic Journal, 102.

FISCHER, S., S. RATNA e C. VÉGH (1996a), "Stabilization and Growth in Transition Economies: The Early Experience," Journal of Economic Perspectives, Summer 1996.

FISCHER, S., S. RATNA e C. VÉGH (1996b), "From Transition to Market: Evidence and Growth Prospects," mimeog., FMI.

GAVIN, M. (1993), "Unemployment and the Economics of Gradualist Reform," mimeog., Columbia University.

KAHNEMAN, D. e A. TVERSKY (1979), "Prospect Theory: an Analysis of Decision Under Risk," Econometrica, Março.

KAHNEMAN, D. e A. TVERSKY (1984), "Choices, Values, and Frames," American Psychologist, Abril.

KAHNEMAN, D., J. KNETSCH, and R. THALER (1990), "Experimental Tests of the Endowment Effect and the Coase Theorem," Journal of Political Economy, 98.

MURRELL, P. (1996), "How Far Has the Transition Progressed?," Journal of Economic Perspectives, Summer 1996.

MUSSA, M. (1982), "Government Policy and the Adjustment Process," in Import Competition and Response, ed. por Jagdish Bhagwati. 
MUSSA, M. (1986), "The Adjustment Process and the Timing of Trade Liberalization," in Economic Liberalization in Developing Countries, ed. por A. M. Choksi e D. Papageorgiou, Basil Blackwell.

RODRIK, D. (1989), "Promises, Promises: Credible Policy Reform via Signalling," The Economic Journal, 99.

RODRIK, D. (1994), "What Does the Political Economy Literature on Trade Policy (Not) Tell Us that We Ought to Know," mimeog., Columbia University.

RODRIK, D. (1995), "Trade and Industrial Policy Reform in Developing Countries: A Review of Recent Theory and Evidence," in Handbook of Development Economics, vol. 3b, J. Behrman \& T. N. Srinivasan (eds.), North-Holland.

THALER, R. (1980), "Toward a Positive Theory of Consumer Choice," Journal of Economic Behavior and Organization, 1.

TOMMASI, M. e A. VELASCO (1995), "Where Are We in the Political Economy of Reform?" mimeog., Columbia University.

TVERSKY, A. and D. KAHNEMAN (1991), "Loss Aversion in Riskless Choice: A Reference-Dependent Model," Quarterly Journal of Economics, Novembro.

WEI, S. J. (1993), "Gradualism versus Big Bang: Speed and Sustainability of Reforms,"

J. F. K. School of Government, Faculty Research Working Paper R93-2. 